\title{
Correction to: Investigating Stranger Things
}

\author{
Tracey Mollet and Lindsey Scott
}

\section{Correction to:}

T. Mollet and L. Scott (eds.), Investigating Stranger Things, https://doi.org/10.1007/978-3-030-66314-8

The original version of the book was inadvertently published without incorporation of the final corrections. The book has been updated with the changes. 